\title{
Resolution improvement for enhancing interference fringes of digital holograms with near infrared illuminance
}

\author{
Ning Liu*, Chao Yang \\ Nanjing University of Posts and Telecommunications, School of Optoelectronics \\ Engineering, Nanjing, Jiangsu 210023, China \\ *Corresponding author: coolboy006@sohu.com
}

\begin{abstract}
A new resolution improving method of enhancing the interference stripes, which can achieve better reconstruction of the recorded object of infrared digital holography has been proposed in this paper. The experiment is conducted under near infrared illuminance. We create poor quality holograms with very low-resolution interference stripes and enhance it with our new method. The processed holograms have much higher-resolution interference stripes. This method is based on interpolation theory, and we make it works fast, stable and easy to apply. Both the forward and backward interpolation of the oriental and portrait direction of the source pixels have been calculated, and have been used to realize the final aim of resolution improvement. During the experiment, since the responsivity of the CCD is not satisfied with the illuminance laser, which is by design, we also give specific analysis on the experimental setup of our work to make sure the object information can be fully recorded by the optical setup but cannot be reconstructed because of the low-resolution and unobvious of the interference stripes. After the resolution improvement process of the original hologram, the object information can then be fully reconstructed, which is very clear in the paper. Figures and dataflow give evidence and demonstrate the good performance of our method.
\end{abstract}

Keywords: infrared digital holography; resolution improvement; interference stripes enhancement; image process.

\section{Introduction}

Digital holography has been considered as an effective way of acquiring the full information of objects, and it has been used in the field of microscopy [1,2], three dimensional imaging [3,4], monitoring [5,6], particle measurement [7,8], and so on. Under most circumstances nowadays, the holograms of digital holography are recorded using a Charged-Couple Device (CCD) instead of the holographic plate for easy calculation with the computers. However, the size of the CCD sensitive array is much smaller than the holographic plate and only the object at a long distance from the array can be recorded due to its low spatial resolution. The reconstruction of the low spatial resolution holograms usually suffers the problem of blur, unclear and loss of details of the original object. These problems may be less obvious when reconstructing the transparent object like cells, but it is much serious when reconstructing the scatter objects. In this case, super-resolution technique has been widely studied to increase the spatial resolution of the holograms for the aim of better reconstructions. Meanwhile, digital holography also possesses drawbacks similar to that of most coherent methods, such as speckle noise arising from the roughness of the object surface when height variations exceed light wavelengths [19,20]. When a coherent light beam 
illuminates the rough surfaces of randomly scattering objects, the waves scattered by different surface points fluctuate statistically because of height variations. The superposition of these scattered waves forms a stationary speckle pattern in the recording plane [21,22,23]. Thus, the hologram not only includes the grating structure of the light filed in object plane, it also incorporates speckles. The presence of speckle noise in reconstructed images degrades the image resolution and measurement accuracy $[24,25]$.

In recent years, many researchers have proposed effective methods to enhance the hologram in order to acquire higher resolution of the reconstruction image. For example, Zhang proposed a non-iterative reconstruction technique by combining multiple shifted low-resolution holographic images to overcome the resolution problem [9], Wang et al. placed a microsphere very close to the object to increase the resolution by transforming the object wave from the higher frequency to the lower one, the resolution enhancement then can be achieved from a single hologram [10], Granero et al. used a diffraction grating between the input object and the CCD recording device to direct additional high-order spatial frequency content of the object spectrum towards the CCD [11], Liu et al. used similar method to achieve this goal [12], Hussain et al. used tilted illumination coupled with object rotation to increase the resolution of the reconstructed image [13], Zhang et al. applied a non-iterative multi-frame registration of the holograms to increase the spatial resolution [14], Jiang et al. placed a random phase plate between the object and the CCD and then using an iterative approach to enhance the resolution of digital holography [15], Latychevskaia et al. used a self-extrapolation approach to pad the surroundings of the hologram and then conduct an iterative reconstruction procedure to enhance the resolution [16], and so on. These methods all did great jobs in enhancing the resolution of the reconstruction. They all concentrated on how to holographical record as much as the higher frequency information of the object, which in order to achieve high resolution reconstruction. As we know, the recoding of object information is related to the character of the interference stripes, such as the tilted angle, density and contrast, and so on. While the quality of the interference stripes is related to the optical setup, the quality of the equipment, laser power and the CCD. All the modern super-resolution methods are focused on creating and capturing more interference stripes to acquired much higher frequency detail of the entire object. If the quality of the interference stripes is degraded, the object cannot be fully retrieved.

Usually under the lab environment, the quality of the interference stripes can easily be guaranteed. But when the digital holographic technology is brought out of the lab into the real world application, the quality of the captured interference stripes will degrade seriously, especially using the laser source within the visible light spectrum. Or sometimes during the experiment, some slightly change of the experimental setup will cause the interference stripes degrade. When these situations happen, it can barely reconstruct the object from the hologram even with the same optical setup or the quality of the equipment, all because of the degradation of the interference stripes. In this paper, we raised a novel, easy approach the enhance the resolution of the interference stripes of the captured hologram, which will give better reconstruction of the object. We adopt a Mach-Zender interference optical setup to conduct our experiment. In order to degrade the quality of the interference stripes, we innovatively use an $850 \mathrm{~nm}$ near infrared illuminance as the light source, and use a CCD that works under visible light spectrum as the detect device. We use a scatter coin as the object in 
the experiment so that the interference stripes captured by the CCD will be very less obvious. When we conduct the reconstruction process, the object cannot be fully retrieved, too much information will be lost. After our resolution improvement process of the hologram, the interference stripes become much clearer, and the quality of the reconstruction is improved significantly.

This resolution improvement process is quite unlike the methods mentioned above. It is basically an interpolation process of a single hologram. It must be very careful that the interpolation cannot ruin the character of the original hologram, if this happens, the reconstruction will fail. Besides, this process focus on single hologram, which need no multi-frame calculation or sophisticated equipment such as microsphere or grating plate. It will greatly improve the computational speed than the multi-frame methods and simplified the experiment than those which need them. Also, it is surely faster than the methods which need iterative process. We calculate the pixel relativity of the original hologram and acquire higher resolution through the merge computation. This method not only increase the use ratio of the information of the source hologram, but also decrease the accumulation of the interpolation error. Most importantly, it can greatly enhance the interference stripes and get the better quality of reconstruction.

This paper is organized as follows: in section 2, the optical experimental setup will be detailed introduced; in section 3, the main strategy of the newly raised resolution improvement method will be detailed introduced; in section 4, we give demonstrate the effort of our method to prove its effectiveness; in section 5, we give conclusion of our method.

\section{Experimental setup}

\subsection{Optical setup}

In this section, we will discuss the optical setup of our experiment, and determine the best location of the CCD and the object. In our experiment, we adopt a Mach-Zender optical path, and use a laser to illuminate a metal coin. The optical setup is shown as follows:

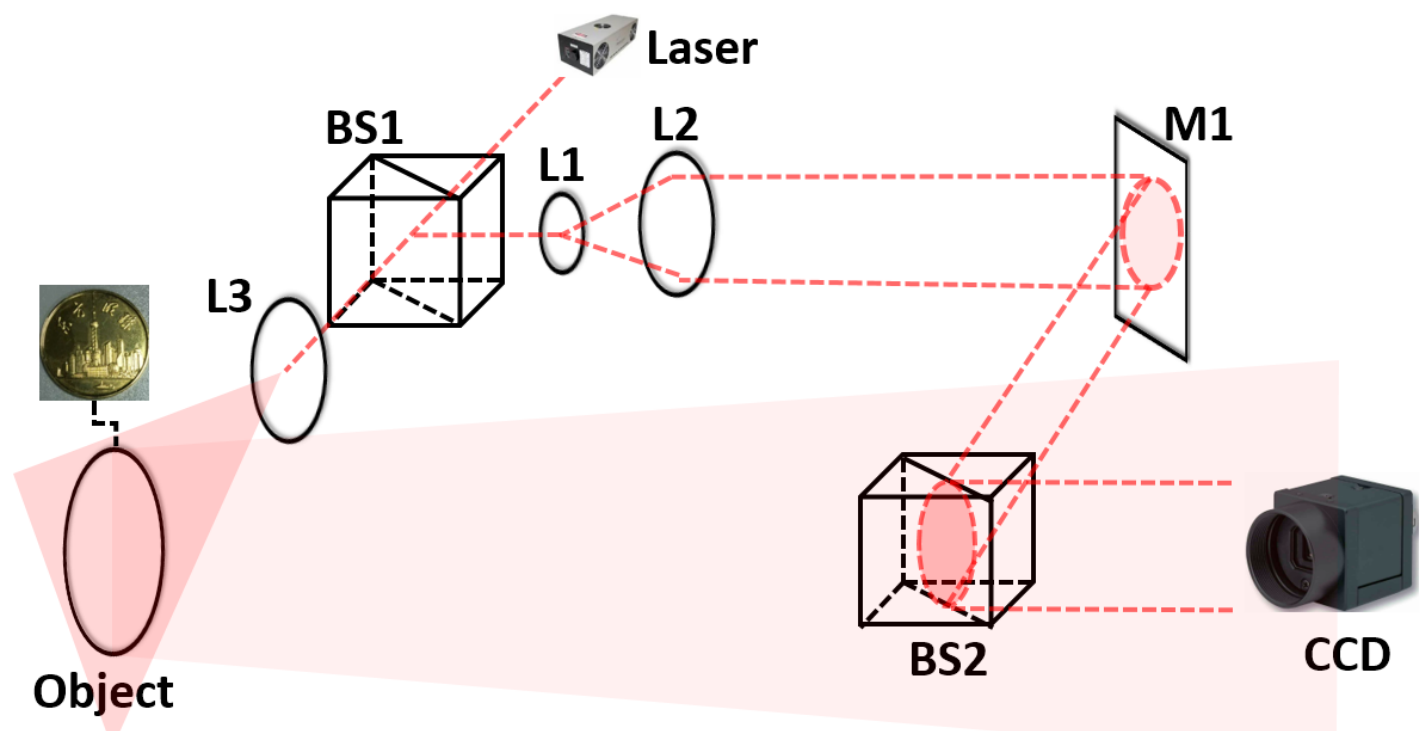

Fig.1 optical setup of our experiment

In our experiment, the laser source we choose is a $32 \mathrm{mw}$ semiconductor laser with the wavelength of $850 \mathrm{~nm}$. The CCD we use is type dmk23u445 of the Imaging Source, it has a 
focal plane array with the dimension of $1280 \times 960$, the pixel distance is $3.75 \mu \mathrm{m}$ in both horizontal and vertical direction. L1, L2, L3 are focal lenses, BS1, BS2 are beam splitters, M1 is a reflective mirror. The object we use is commemorative coin with the diameter of $65 \mathrm{~mm}$. The reflective wave from the coin is consider as the object wave, while the reflective wave from M1 is considered as the reference wave in our experiment. This CCD has a very good spectrum response at the wavelength of $632.8 \mathrm{~nm}$, but when it comes to the wavelength of $850 \mathrm{~nm}$, the spectrum response curve drops fast, only about $40 \%$ left. The spectrum response curve of this CCD is shown in fig.2. The reason we choose this kind of laser and CCD combination is that, first we set the CCD and the object in a proper condition, which will be discussed in the next section. This is for creating the best interference stripes of the object wave and the reference wave. Then, considering that the CCD is not responding well at the chosen wave length, the interference stripes will become very weak and hardly to observe, but they still exist. Thus, we can create a hologram with very low resolution of the interference stripes, when the reconstruction process is conducted, although the object should be fully recorded according to the optical setup theoretically, it fails anyway due to the low resolution of the interference stripes. After we apply our resolution improvement process onto the original low resolution hologram, the stripes will be enhanced significantly, and the full object information will be reconstructed.

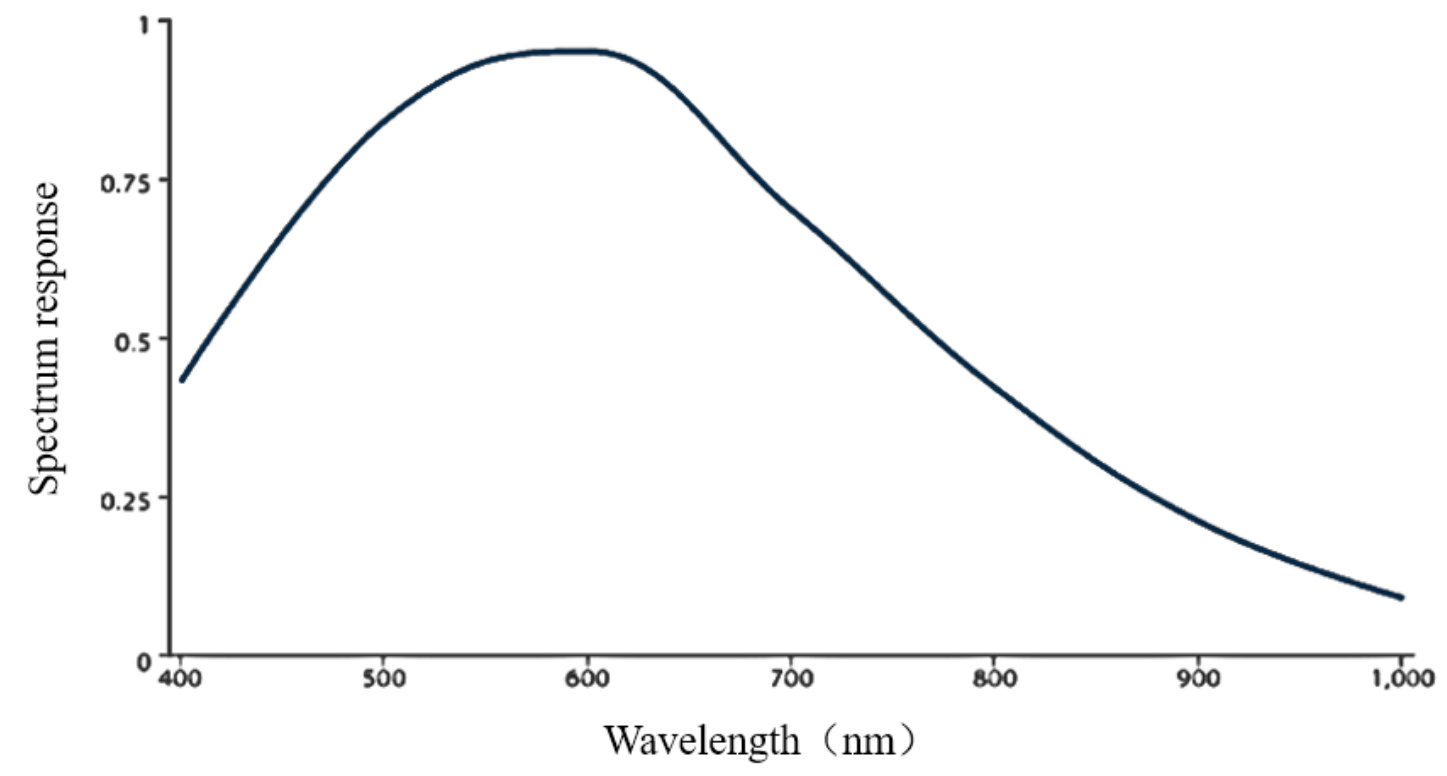

Fig.2 spectrum response of the CCD

\subsection{Detection study of the CCD and the object}

As we mentioned above, the wavelength of the laser is not quite satisfied with the spectrum response of the CCD. We use this strategy to lower the resolution of the hologram generated by the interference of the object wave and the reference wave. We can create a hologram which contains the full object information but cannot be fully reconstructed due to the unobvious of the stripes. To achieve this, the placement of the CCD and the object needs to be well arranged. In our experiment, the width of the CCD focal plane is $\mathrm{L}$, the diameter of the object is $\mathrm{D}_{0}$, the distance between the CCD and the object is $\mathrm{d}$. The digital holographic recording scheme is shown as follows: 


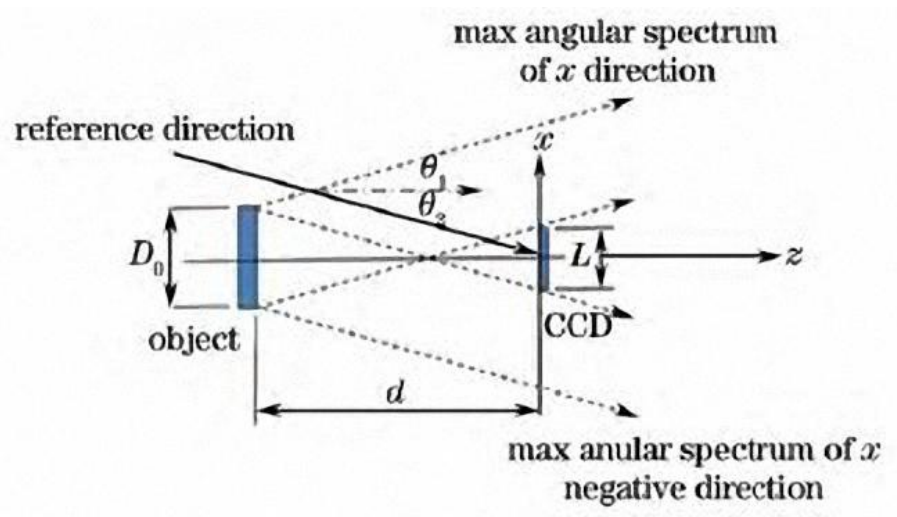

Fig.3 Digital holographic recording scheme of the CCD

Let us consider the object wave as $O_{0}(x, y)$, the wave field propagated to the CCD along the $\mathrm{z}$ axis can be expressed as eq. 1 according to the angular diffraction theory:

$$
O(x, y)=F^{-1}\left\{F\left[O_{0}(x, y)\right] \exp \left[j \frac{2 \pi}{\lambda} d \sqrt{1-\left(\lambda f_{x}\right)^{2}-\left(\lambda f_{y}\right)^{2}}\right]\right\}
$$

Where

$$
F\left[O_{0}(x, y)\right]=\int_{-\infty}^{\infty} \int_{-\infty}^{\infty} O_{0}(x, y) \exp \left[-j 2 \pi\left(f_{x} x+f_{y} y\right)\right] d x d y
$$

$j=\sqrt{-1}, k=2 \pi / \lambda$ represents the wave number, $\lambda$ is the wavelength of the laser, $f_{x}, f_{y}$ are the frequency coordinates of $\mathrm{x}, \mathrm{y}$. The object wave field can be expressed as:

$$
O_{0}(x, y)=\int_{-\infty}^{\infty} \int_{-\infty}^{\infty} F\left[O_{0}(x, y)\right] \exp \left[j \frac{2 \pi}{\lambda}\left(\lambda f_{x} x+\lambda f_{y} y\right)\right] d f_{x} f_{y}
$$

It can be seen from eq. 3 that, the wave field emitted from the object plane can be recognized as the superposition of plane waves. Since CCD has the width limit of the focal plane, the dash arrow represents the propagating direction of the highest angular spectrum of the object wave, $\theta_{1}$ represents the angle between the $\mathrm{x}$ axis and the $\mathrm{z}$ axis of the highest angular spectrum of object wave which propagates towards $\mathrm{z}$ axis. If the pixel distance of the CCD is $\Delta x$, according to the sampling theory, the minimum width of the recordable interference stripes is $2 \Delta x$. According to fig. 1 , the reference wave is a plane wave, the angle between the propagating direction of reference wave and the $\mathrm{z}$ axis is $\theta_{2}$. Let $A_{1} \exp \left(j k x \sin \theta_{1}\right)$ represents the plane wave with the highest angular spectrum, $A_{2} \exp \left(-j k x \sin \theta_{2}\right)$ represents the reference wave, thus, the amplitude interference of these two waves can be expressed as:

$$
I(x)=A_{1}^{2}+A_{2}^{2}+2 A_{1} A_{2} \cos \left[k x\left(\sin \theta_{1}+\sin \theta_{2}\right)\right]
$$


Under the paraxial approximation, $\sin \theta_{1}=\theta_{1}=\frac{L+D_{0}}{2 d}, \sin \theta_{2}=\theta_{2}$, so the width of interference stripes is determined as:

$$
T=\frac{\lambda}{\frac{L+D_{0}}{2 d}+\theta_{2}}
$$

Given $T=2 \Delta x$, we can get from eq. 5 that:

$$
\frac{L+D_{0}}{2 d}+\theta_{2}=\frac{\lambda}{2 \Delta x}
$$

In order to fully separate the \pm 1 spectrum when conducting the Fast Fourier Transform (FFT) onto the captured hologram, the angle between the reference wave and the $\mathrm{z}$ axis should be fulfilling the condition of $\theta_{2}=\frac{3 \times 4 D_{0}}{8 d}$ [17], thus, we have:

$$
\frac{L+D_{0}}{2 d}+\frac{12 D_{0}}{8 d}=\frac{\lambda}{2 \Delta x}
$$

From eq.7 we can see that, the best recording distance which could fully take advantage of the performance of the CCD in off-axis digital holography is calculated by:

$$
d=\left(L+4 D_{0}\right) \Delta x / \lambda
$$

Next, let us discuss the equivalent width of the CCD. Once the distance has been established, the equivalent width then determines the resolution limit of the optical system. According to eq.3, the highest angular frequency $f_{x}$ which could be recorded by the CCD has to fulfill the next equation:

$$
\lambda f_{x}=\theta_{1}=\frac{L+D_{0}}{2 d}
$$

Because the maximum recordable frequency of the CCD is $f_{\max }=\frac{1}{2 \Delta x}$, according to eq.9, the equivalent width of the CCD $L_{e}$ when conducting the resolution improvement recording should fulfill the condition as follows:

$$
\frac{1}{2 \Delta x}=\frac{L_{e}+D_{0}}{2 \lambda d}
$$

That is to say, the equivalent width $L_{e}$ can be expressed as: 


$$
L_{e}=\frac{\lambda d}{\Delta x}-D_{0}
$$

From the above deduction we can see that, when the parameters in fig. 3 are determined, the equivalent with of the CCD $L_{e}$ is then determined. If the object size is bigger than $L_{e}$, the CCD cannot record higher angular frequency of the object wave, which will cause ill reconstruction of the object, meanwhile, the performance of the CCD cannot be fully developed if the optical setup is smaller than $L_{e}$, the theoretical calculation must be the same as the real optical setup. In our experiment, we setup the optical path according to which discussed above and make sure that the object information will be mostly recorded by the CCD. The only problem is that the wavelength of the laser will not cause enough response by the $\mathrm{CCD}$, the interference stripes will be very unobvious which leads to a bad reconstruction of the object. If we can greatly super-enhance the stripes, we can still get a good reconstruction of the object.

\section{Super-resolution method of the interference stripes}

In this section, we will discuss the main strategy of our resolution improvement method of enhancing the interference stripes. Unlike the usual common super-resolution methods of digital holography, our main focus is to super-enhance the unobvious interference stripes of the hologram for reconstructing all the object information. That means, the super-resolution process cannot destroy the original interference pattern of the hologram which may cause the failure of the upcoming reconstruction. For this consideration, we design an improved Newton interpolation method. The whole process has the advantage of easy calculation, fast and stable. It will be discussed step by step.

The main resolution improvement interpolation scheme is shown as fig.4, which gives the interpolation of the source pixels and the target (interpolation) pixels.

\begin{tabular}{|c|c|c|c|c|c|c|c|}
\hline & $\mathrm{i}$ & $\mathrm{m}$ & $\mathrm{i}+1$ & $\mathrm{~m}+1$ & $\mathrm{i}+2$ & $\mathrm{~m}+3$ & $\mathrm{i}+3$ \\
\hline $\mathrm{j}$ & $\bigcirc$ & $\bullet$ & $\bigcirc$ & $\bullet$ & $\bigcirc$ & $\bullet$ & $\bigcirc$ \\
\hline $\mathrm{k}$ & $\bullet$ & $\odot$ & $\bullet$ & $\odot$ & $\bullet$ & $\odot$ & $\bullet$ \\
\hline $\mathrm{j}+1$ & $\bigcirc$ & $\bullet$ & $\bigcirc$ & $\bullet$ & $\bigcirc$ & $\bullet$ & $\bigcirc$ \\
\hline $\mathrm{k}+1$ & $\bullet$ & $\odot$ & $\bullet$ & $\odot$ & $\bullet$ & $\odot$ & $\bullet$ \\
\hline $\mathrm{j}+2$ & $\bigcirc$ & $\bullet$ & $\bigcirc$ & $\bullet$ & $\bigcirc$ & $\bullet$ & $\bigcirc$ \\
\hline $\mathrm{k}+2$ & $\bullet$ & $\odot$ & $\bullet$ & $\odot$ & $\bullet$ & $\odot$ & $\bullet$ \\
\hline $\mathrm{j}+3$ & $\bigcirc$ & $\bullet$ & $\bigcirc$ & $\bullet$ & $\bigcirc$ & $\bullet$ & $\bigcirc$ \\
\hline
\end{tabular}

Fig.4 relative position between source pixel and target pixel

In fig.1, the hollow circle represents the source pixels, the solid circle and the dot circle represent the target pixels. In order to realize the resolution improvement of the interference stripes, we use the value of the source pixels to calculate the interpolation result from both horizontal and vertical direction. Then, we calculate the resolution improvement interpolation results based on the potential pixel relativeness by means of the merge computation.

According to fig.4, first we calculate the interpolation result of $f_{j, m+1}$. To do this, we use 
the pixels of $f_{j, i}, f_{j, i+1}$ and $f_{j, i+2}$ to calculate the oriental forward interpolation, and get the result marked $f_{j, m+1}^{\prime}$. Then we use the pixels of $f_{j, i+3}, f_{j, i+2}$ and $f_{j, i+1}$ to calculate the oriental backward interpolation, and get the result marked $f_{j, m+1}^{\prime \prime}$. The resolution improved pixel value of $f_{j, m+1}$ can be expressed as:

$$
f_{j, m+1}=w_{1} f_{j, m+1}^{\prime}+w_{2} f_{j, m+1}^{\prime \prime}
$$

Where w1 and w2 represent the relativeness weighting coefficient of forward and backward interpolation. These coefficients give the relativeness of the source pixel when conducting the interpolation. The relativeness depicts the property of the image area where the source pixels have been used during the interpolation. The larger consistency of the property, the bigger the relativeness is, furthermore, the bigger the weighting coefficient is. That means, the relativeness of the source pixel can be calculated by the second order differentiation:

$$
\begin{gathered}
\Delta^{2} f_{+}=\left|\left(f_{j, i+2}-f_{j, i+1}\right)-\left(f_{j, i+1}-f_{j, i}\right)\right|=\left|f_{j, i+2}-2 f_{j, i+1}+f_{j, i}\right| \\
\Delta^{2} f_{-}=\left|\left(f_{j, i+1}-f_{j, i+2}\right)-\left(f_{j, i+2}-f_{j, i+3}\right)\right|=\left|f_{j, i+1}-2 f_{j, i+2}+f_{j, i+3}\right|
\end{gathered}
$$

The relativeness weighting coefficient can be expressed as:

$$
\begin{aligned}
& W_{1}=\frac{\Delta^{2} f_{+}}{\Delta^{2} f_{+}+\Delta^{2} f_{-}} \\
& W_{2}=\frac{\Delta^{2} f_{1}}{\Delta^{2} f_{+}+\Delta^{2} f_{-}}
\end{aligned}
$$

Similarly, for pixel $f_{k+1, i}$, its portrait forward and backward interpolation of $f_{k+1, i}^{\prime}$ and $f_{k+1, i}^{\prime \prime}$ can be calculated by pixel $f_{j, i}, f_{j+1, i}, f_{j+2, i}, f_{j+3, i}$, and the resolution improvement interpolation of $f_{k+1, i}$ can then be calculated by adding proper relativeness weighting coefficient, respectively.

In our experiment, we calculate the pixel $f_{k+1, m+1}$ within the resolution improved image by the forward and backward weighting interpolation of the source pixel which has the orientation of $45^{\circ}$ or $135^{\circ}$ :

$$
\begin{aligned}
f_{k+1, m+1} & =w_{1}^{45^{\circ}} f_{k+1, m+1}^{\prime 45^{\circ}}+w_{2}^{45^{\circ}} f_{k+1, m+1}^{\prime \prime 45^{\circ}}+w_{1}^{135^{\circ}} f_{k+1, m+1}^{\prime 135^{\circ}}+w_{2}^{135^{\circ}} f_{k+1, m+1}^{\prime \prime 135^{\circ}} \\
& \Delta^{2} f_{+}^{45^{\circ}}=\left|f_{j+3, i}-2 f_{j+2, i+1}+f_{j+1, i+2}\right| \\
& \Delta^{2} f_{-}^{45^{\circ}}=\left|f_{j, i+3}-2 f_{j+1, i+2}+f_{j+2, i+1}\right|
\end{aligned}
$$




$$
\begin{aligned}
& \Delta^{2} f_{+}^{135^{\circ}}=\left|f_{j+3, i+3}-2 f_{j+2, i+2}+f_{j+1, i+1}\right| \\
& \Delta^{2} f_{-}^{135^{\circ}}=\left|f_{j, i}-2 f_{j+1, i+1}+f_{j+2, i+2}\right| \\
& W_{1}^{45^{\circ}}=\frac{\Delta^{2} f_{+}^{45^{\circ}}}{\Delta^{2} f_{+}^{45^{\circ}}+\Delta^{2} f_{-}^{45^{\circ}}+\Delta^{2} f_{+}^{135^{\circ}}+\Delta^{2} f_{-}^{135^{\circ}}} \\
& w_{2}^{45^{\circ}}=\frac{\Delta^{2} f_{-}^{45^{\circ}}}{\Delta^{2} f_{+}^{45^{\circ}}+\Delta^{2} f_{-}^{45^{\circ}}+\Delta^{2} f_{+}^{135^{\circ}}+\Delta^{2} f_{-}^{135^{\circ}}} \\
& W_{1}^{135^{\circ}}=\frac{\Delta^{2} f_{+}^{135^{\circ}}}{\Delta^{2} f_{+}^{45^{\circ}}+\Delta^{2} f_{-}^{45^{\circ}}+\Delta^{2} f_{+}^{135^{\circ}}+\Delta^{2} f_{-}^{135^{\circ}}} \\
& W_{2}^{135^{\circ}}=\frac{\Delta^{2} f_{-}^{135^{\circ}}}{\Delta^{2} f_{+}^{45^{\circ}}+\Delta^{2} f_{-}^{45^{\circ}}+\Delta^{2} f_{+}^{135^{\circ}}+\Delta^{2} f_{-}^{135^{\circ}}}
\end{aligned}
$$

Where, $f_{k+1, m+1}^{\prime 45^{\circ}}, f_{k+1, m+1}^{\prime \prime 45^{\circ}}, f_{k+1, m+1}^{\prime 135^{\circ}}$ and $f_{k+1, m+1}^{\prime \prime 135^{\circ}}$ represent the interpolation of forward $45^{\circ}$, backward $45^{\circ}$, forward $135^{\circ}$, and backward $135^{\circ}$. Because all the resolution improved pixels' value is calculated through the weighting interpolation of the source pixel of original hologram, the accumulation error can then be reduced, which improves the quality of the resolution improved image. By applying this method onto the original hologram, the interference stripes can be greatly enhanced.

\section{Analysis of the experimental results}

In this section, we will analyze the performance of this newly raised resolution improvement method. The computational laptop we use has an inter(R) Core(TM) i5-6300U CPU with the frequency of $2.40 \mathrm{GHz}$, it also has an $8 \mathrm{~GB}$ DDR4 ram. First, we give figure demonstration to show the effectiveness of our method.

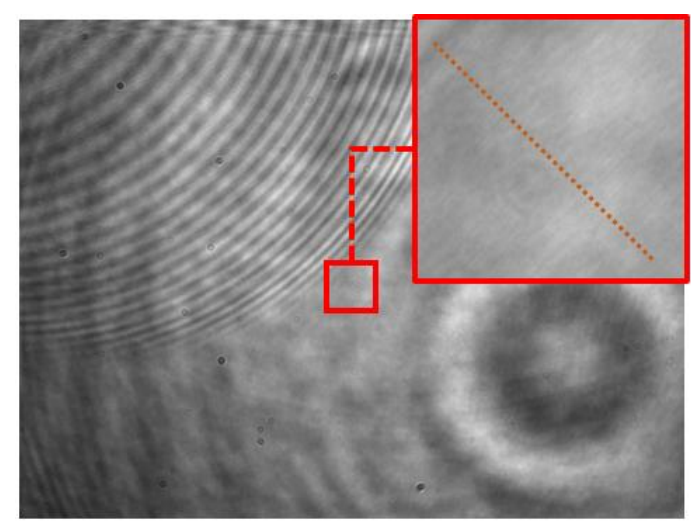

(a)

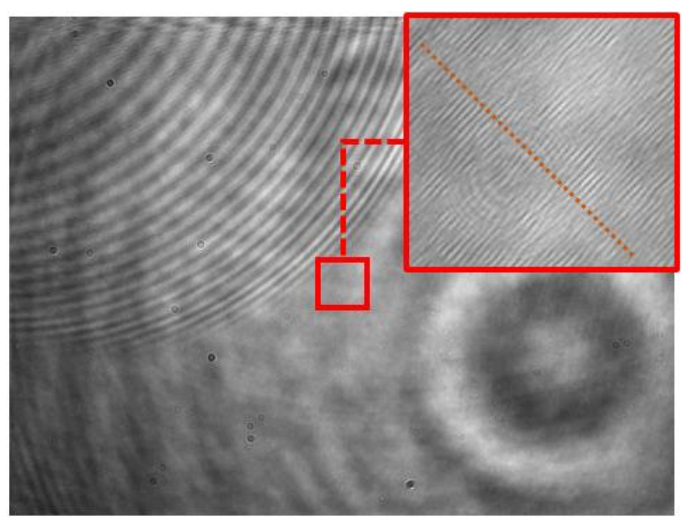

(b)

Fig.5 resolution improvement effectiveness of the proposed method. (a) the original hologram. (b)the super-enhanced hologram

Fig.5 (a) is the original hologram. As it can be seen, the interference stripes are very unobvious in the original hologram. As we mentioned above, this is because that the CCD can only reach about $40 \%$ responsivity of the $850 \mathrm{~nm}$ near infrared laser illuminance. After the 
resolution improvement process, the interference stripes are very well enhanced. Here we explain that, the common super-resolution methods, such as multi-illuminance method, multi-frame iteration and so on, are mainly focused on increasing the density of the interference stripes to make the reconstruction object much clearer. Our method, however unlike the common methods, is focused on enhancing the quality of the interference stripes, which can be used to fully reconstruct the object information. Also, it can be seen from fig.5 that, our method does not change or destruct the shape and distribution of the interference stripes, which is promising to succeed in object information reconstruction without adding or erasing it.

Next, we use fig. 5 to conduct the reconstruction process separately. The reconstruction process is according to eq.1. We use the angular spectrum reconstruction instead of the Fresnel reconstruction. The result is shown as follows:

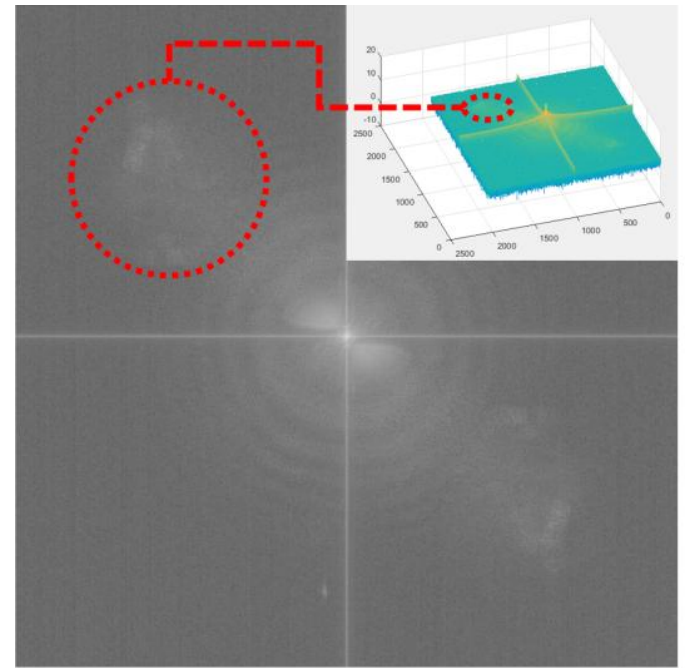

(a)

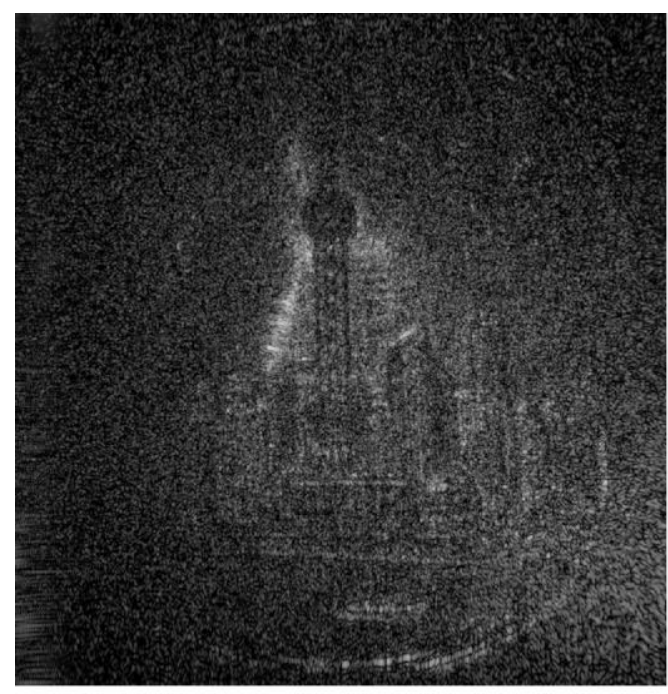

(c)

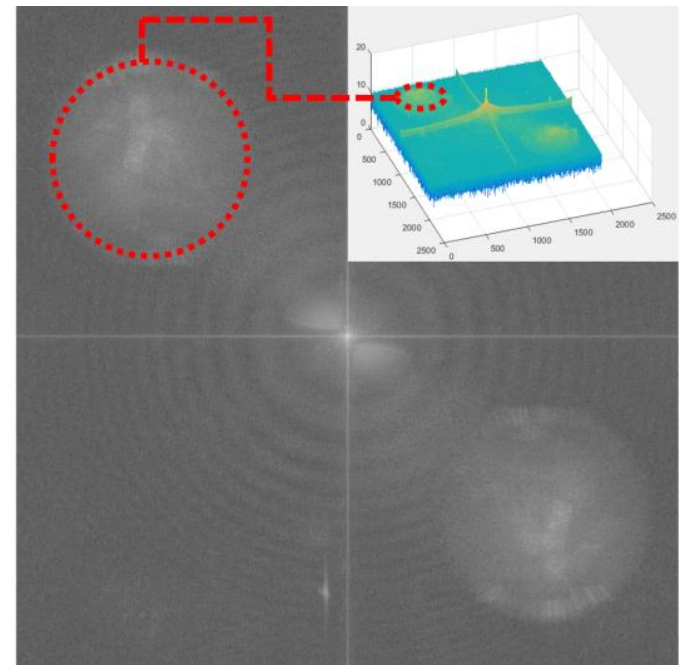

(b)

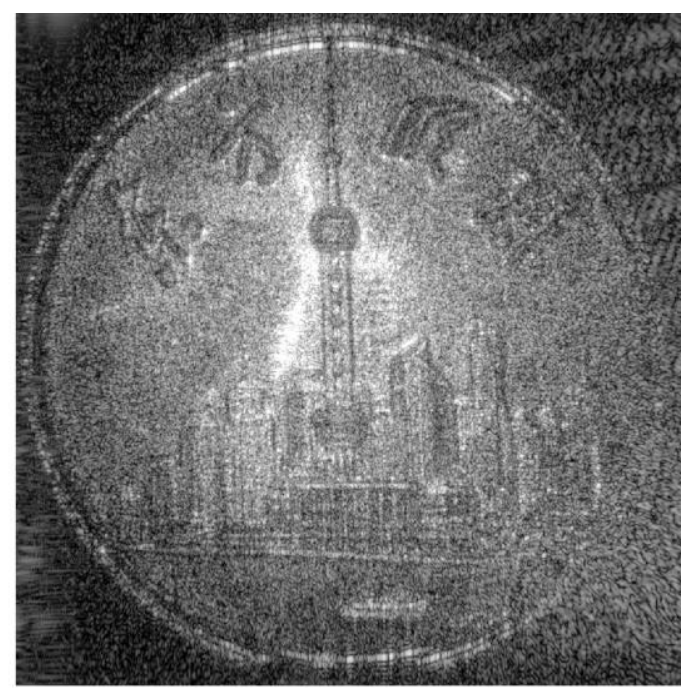

(d)

Fig.6 the comparison of the reconstruction of object information. (a)the FFT of the original hologram. (b) the FFT of the super-enhanced hologram. (c) the object reconstruction of the original hologram. (d)the object reconstruction of the super-enhanced hologram.

We conduct the FFT on the original low-resolution hologram and the processed 
high-resolution hologram and get the results shown in fig.6. As it can be seen in fig.6(a), the \pm 1 order frequency spectrum of the object can hardly be observed in the frequency domain. The reconstruction of hologram with low-resolution interference stripes can only retrieve very little object information as in fig.6(c). Only the central pattern of the coin can be reconstructed, the four Chinese character on the top part of the coin has been missed miserably. The contour of the coin is also cannot be displayed. Meanwhile, even the reconstructed information of the object is hard to tell what it is. While in fig.6(b), the \pm 1 order frequency spectrum of the object is very clear in the frequency domain. The coin information has been fully retrieved by the processed hologram with high-resolution of the interference stripes. Also, since the interference stripes have been enhanced, the amplitude of the \pm 1 order has been enhanced either. This is the reason that fig.6(d) looks much brighter than fig.6(c). During the computation in Matlab, we set the display range of the reconstruction at the same scale so that the brightness improvement is based on the enhancement of the interference stripes.

Next, we give other figure demonstration of the performance of our method. Fig.7 is a pair of holograms with and without our method. The object is a reflective MEMS chip.

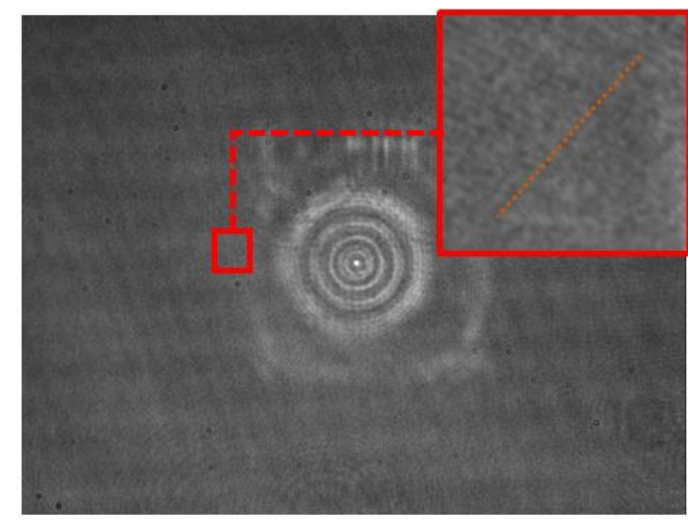

(a)

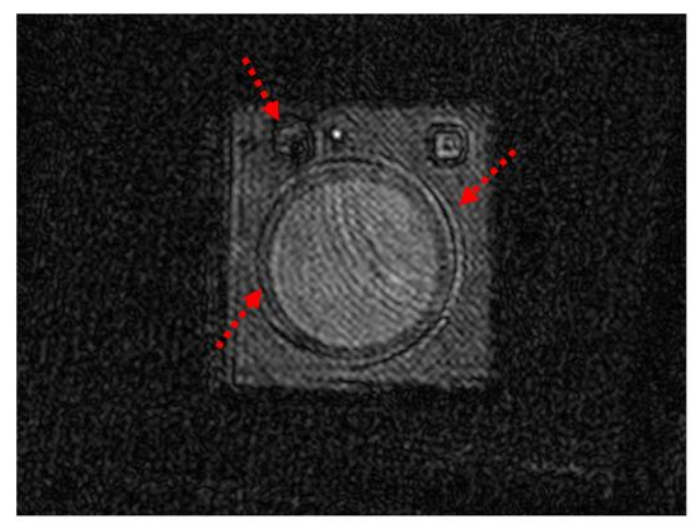

(c)

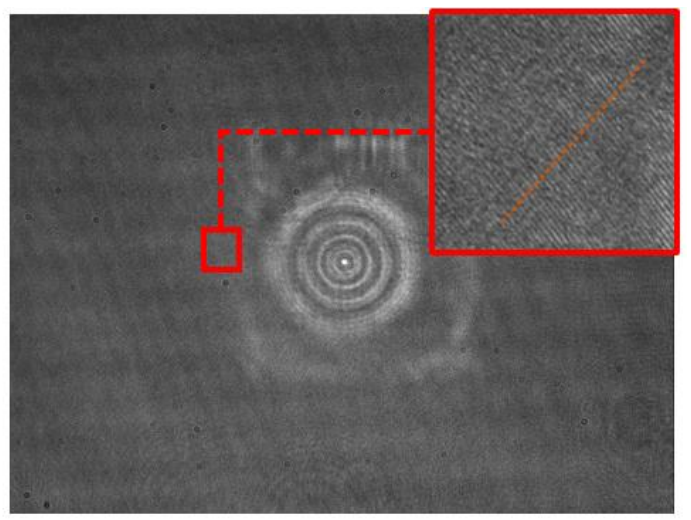

(b)

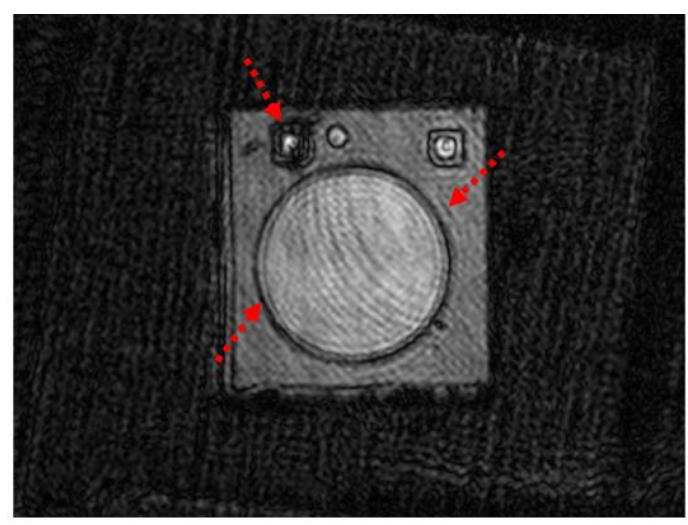

(d)

Fig.7 the comparison of holograms and the numerical reconstruction of a MEMS object with and without the proposed method. (a) original low-resolution hologram. (b) processed resolution improved hologram. (c)reconstruction of low-resolution hologram. (d)reconstruction of resolution improved hologram.

As we can see from fig.7(a), the interference stripes on the original hologram are very unobvious and hard to observe according to the big red square. Meanwhile, the reconstruction 
of the low-resolution hologram is blurry, some object information is also missed. However, in fig.7(b), the resolution of the interference stripes has been greatly improved, and can easily be observed. The quality of the reconstruction is also much better than fig.7(c). Here we have to explain the reason why the reconstruction of the original hologram is better than which shows in fig.5. This because that the coin is a scatter object, it generates the diffuse reflection when the expanded laser beam illuminated on its surface. While the MEMS chip is a polished object which generates the mirror reflection. Meanwhile, the size of the coin is much bigger than the MEMS chip since the dimension of the chip is only $10 \mathrm{~mm} \times 10 \mathrm{~mm}$. In these cases, the light reflects from the coin surface is way much weaker than from the chip surface. Since the laser illuminance is not so satisfied with the CCD, the performance of object reconstruction of the original hologram of fig.5 is much poorer than which of fig.7.

Hence, we calculate the numerical intensity distribution [18] of the selected window to depict the resolution enhancement of our method. As in fig.5 (a)(b) and fig.7(a)(b), the selected windows have the dimension of $40 \times 40$ pixels. The numerical intensity distributions have been calculated toward the direction of the brown line in these selected windows. The results have been demonstrated below.

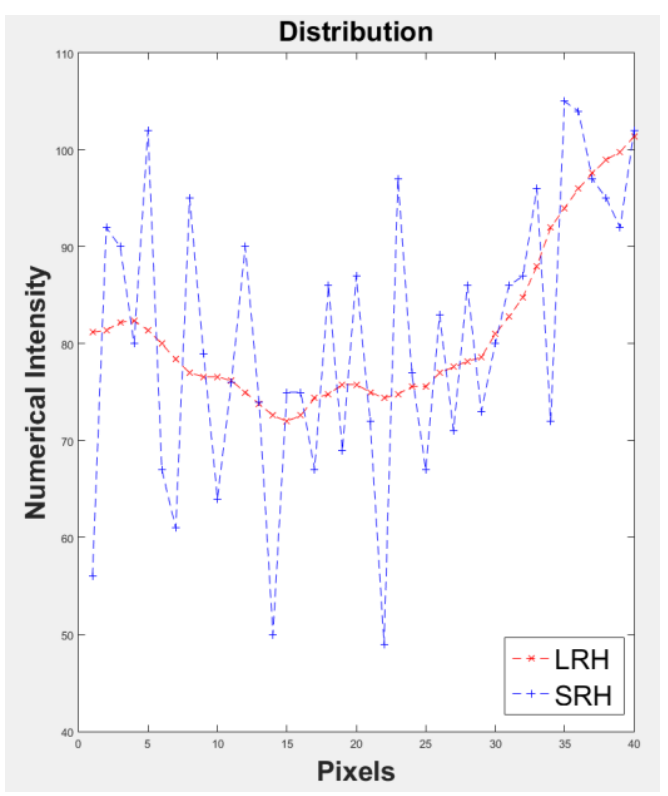

(a)

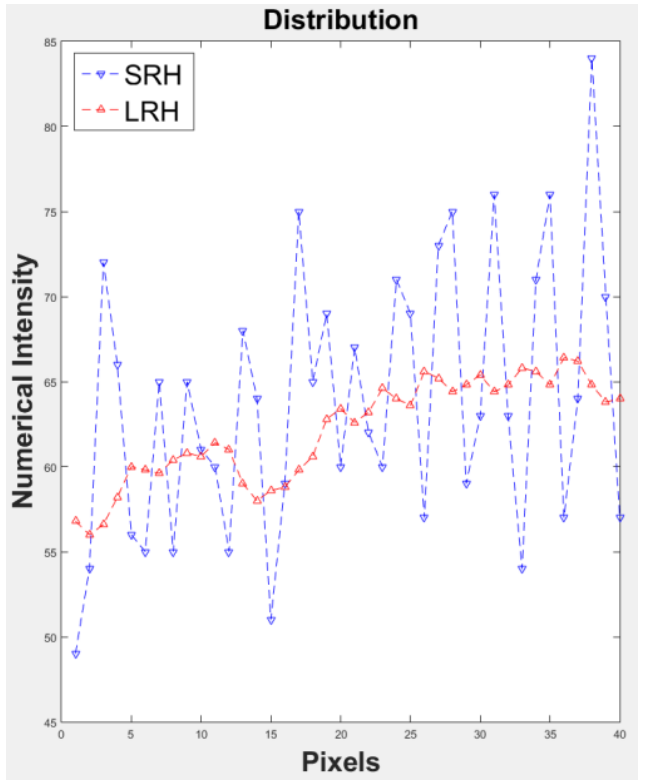

(b)

Fig.8 the numerical intensity distribution of the selected window of fig.5 and fig.7. (a) the intensity distribution plots of fig.5(a) and fig.5(b), the red mark stands for the low-resolution hologram (LRH), the blue mark stands for the super-resolution hologram (SRH). (b) the intensity distribution plots of fig.7(a) and fig.7(b), the red mark stands for the low-resolution hologram (LRH), the blue mark stands for the super-resolution hologram (SRH).

From fig.8(a) we can see that, the intensity distributions of the original holograms are hard to distinguish, while in fig.8(b), the distributions are much distinguishable due to the resolution enhancement of the interference stripes. The improvement of the intensity distribution can also be considered that the spatial resolution has been increased as well. Meanwhile, according to fig.8, we can also deduct that the spatial frequency of the interference stripes has been increased since the pixel intensity distribution has become much steeper. We give the index of spatial frequency (SF) to prove the discussion. The SF index 
reflects the total activity of the pixel value of an image. It contains two parts of spatial row frequency $(\mathrm{RF})$ and spatial column frequency $(\mathrm{CF})$, the calculation of these two parts are expressed as follows:

$$
\begin{aligned}
& R F=\sqrt{\frac{1}{M \times N} \sum_{i=1}^{M} \sum_{j=2}^{N}\left(f_{i, j}-f_{i, j-1}\right)^{2}} \\
& C F=\sqrt{\frac{1}{M \times N} \sum_{i=2}^{M} \sum_{j=1}^{N}\left(f_{i, j}-f_{i-1, j}\right)^{2}} \\
& S F=\sqrt{R F^{2}+C F^{2}}
\end{aligned}
$$

Where $\mathrm{M}, \mathrm{N}$ are the dimension of the hologram. We use eq.26-eq.28 to calculate the data reported in fig.5(a)(b) and fig.7(a)(b), the results are shown in table 1.

Table. 1 the SF comparison of the original holograms and the enhanced holograms

\begin{tabular}{|c|c|c|c|c|}
\hline & \multicolumn{2}{c|}{ Fig.5 } & Fig.7 \\
\hline RF & (a) & (b) & (a) & (b) \\
\hline CF & 4.7382 & 30.8720 & 6.2111 & 28.9452 \\
\hline SF & 4.7114 & 30.4404 & 6.0133 & 25.7912 \\
\hline
\end{tabular}

Table 1 gives a clear demonstration of the enhancement of interference stripes. As we can see, all the three index have been greatly improved when our new method has been conducted onto the original holograms. The SF index goes up to 7 times higher than the original one at most, which shows the same proof as the figure illustration. Our method has great ability of enhancing the low-resolution holograms into their resolution improved version.

This technique can greatly enhance the resolution of the original captured hologram, it can increase the visibility of the interference fringes which contains the object information, and thus retrieve the high quality reconstruction of the original object. Unlike the other super-resolution techniques, this technique is much simpler in calculation and uses no specific optical setup. In the meantime, the other super-resolution techniques can only be effected when the interference fringes are clear, while our technique can effectively make the interference fringes clearer on the hologram level.

\section{Conclusion}

In this paper, we proposed a novel resolution improvement method to enhance the interference stripes. Unlike the common super-resolution methods in digital holography, this method can deal with the very weak and unobvious interference stripes. In our experiment, we set a special recording scheme by lowering the responsivity of the CCD by using the $850 \mathrm{~nm}$ near infrared laser illuminance, which in order to create the very low-resolution holograms to test our method. The experimental results shown that, this method will neither change nor destruct the object information in the hologram, and has the advantage of fast calculating, easy programming and great effectiveness. We believe that this method will work well in the real world digital holographic applications.

\section{Acknowledgements}

This work was supported in part by the National Science Foundation of China under Grant 
61505083 and the Scientific Research Foundation of Nanjing University of Posts and Telecommunications (NO. NY215043).

\section{References}

1. X. Yu, M. Cross, C. Liu, D. C. Clark, D. T. Haynie, and M. K. Kim, "Measurement of the traction force of biological cells by digital holography," Biomed. Opt. Express 3(1), 153-159 (2012).

2. M. Schnell, P. S. Carney, and R. Hillenbrand, "Synthetic optical holography for rapid nanoimaging," Nat.Commun. 5, 3499 (2014).

3. L. Xu, X. Peng, J. Miao, and A. K. Asundi, "Studies of digital microscopic holography with applications to microstructure testing," Appl. Opt. 40(28), 5046-5051 (2001).

4. P. Ferraro, S. Grilli, D. Alfieri, S. De Nicola, A. Finizio, G. Pierattini, B. Javidi, G. Coppola, and V. Striano, "Extended focused image in microscopy by digital Holography," Opt. Express 13(18), 6738-6749 (2005).

5. M. Locatelli, E. Pugliese, M. Paturzo, V. Bianco, A. Finizio, A. Pelagotti, P. Poggi, L. Miccio, R. Meucci, and P.Ferraro, "Imaging live humans through smoke and flames using far-infrared digital holography," Opt. Express 21(5), 5379-5390 (2013).

6. V. Mico, Z. Zalevsky, P. García-Martínez, and J. García, "Superresolved imaging in digital holography by superposition of tilted wavefronts," Appl. Opt. 45(5), 822-828 (2006).

7. F. Dubois, N. Callens, C. Yourassowsky, M. Hoyos, P. Kurowski, and O. Monnom, "Digital holographic microscopy with reduced spatial coherence for three-dimensional particle flow analysis," Appl. Opt. 45(5), 864-871 (2006).

8. Y. Park, G. Popescu, K. Badizadegan, R. R. Dasari, and M. S. Feld, "Fresnel particle tracing in three dimensions using diffraction phase microscopy," Opt. Lett. 32(7), 811-813 (2007).

9. S. Zhang, "Digital hologram resolution enhancement using a fast reconstruction algorithm", Optics Communications, 332, 158-163(2014).

10. Y. Wang, S. Guo, D. Wang, Q. Lin, L. Rong, J. Zhao, "Resolution enhancement phase-contrast imaging by microsphere digital holography", Optics Communications 366,81-87(2016).

11. L. Granero, V. Mico, Z. Zalevsky, J. Garcia, "Superresolution imaging method using phase-shifting digital lensless Fourier holography", Optic Express, 17(17), 15008-15022(2009).

12. C. Liu, Z. Liu, F. Bo, Y. Wang, J. Zhu, "Super-resolution digital holographic imaging method", Applied Physics Letters, 81(17), 3143-3145(2002).

13. A. Hussain, A.A. Mudassar, "Optical super resolution using tilted illumination coupled with object rotation", Optics Communications, 339, 34-40(2015).

14. S. Zhang, J. Zhou, "Image resolution enhancement in digital holography", $10^{\text {th }}$ international conference on natural computation (2014).

15. Z. Jiang, S.P. Veetil, J. Cheng, C. Liu, L. Wang, J. Zhu, "High-resolution digital holography with the aid of coherent diffraction imaging", Optics Express, 23(16),20916-20925(2015).

16. T. Latychevskaia, H.W. Fink, "Resolution enhancement in digital holography by self-extrapolation of holograms", Optics Express, 21(6), 7726-7733(2013). 
17. P. Picart, J. Leval, "General theoretical formulation of image fornation in digital Fresnel holography”, J Opt Soc Am A, 25(7),1744-1761(2008).

18.Y. Kashter, J.Rosen, "Enhanced-resolution using modified configuration of Fresnel incoherent holographic recorder with synthetic aperture", Optics Express, 22(17), 20551-20565,(2014).

19. V. Bianco, P. Memmolo, M. Paturzo, A. Finizio, B. Javidi and P. Ferraro. "Quasi noise-free Digital Holography," Light, Science and Application (2016) 5, e16142

20.V. Bianco, M. Paturzo, P. Memmolo, A. Finizio, P. Ferraro, B. Javidi "Random resampling masks: a non-Bayesian one-shot strategy for noise reduction in digital holography," Opt. Lett. 38(5), 619-621, 2013.

21. P. Memmolo, V. Bianco, M. Paturzo, B. Javidi, P.A.Netti, P. Ferraro. "Encoding multiple holograms for speckle-noise reduction in optical display," Opt. Exp. 22(21), 25768-25775, 2014.

22.Y. S. Kim, T. Kim, S.S. Woo, H. Kang, T.C. Poon, C.Zhou. "Speckle-free digital holographic recording of a diffusely reflecting object", Opt. Exp., 21(7), 8183-8189, 2013.

23. S. Montresor and P. Picart, "Quantitative appraisal for noise reduction in digital holographic phase imaging," Optics Express, 24, 14322-14343 (2016)

24. P. Ferraro, C.D. Core, L.Miccio, S.Grilli, S.D.Nicola, A.Finizio, G.Coppola, "Phase map retrieval in digital holography: avoiding the under-sampling effect by a lateral shear approach" Optics letters 32 (15), 2233-2235 (2007)

25. M. Paturzo, A. Pelagotti, A. Finizio, L. Miccio, M. Locatelli, A. Gertrude, P. Poggi, R. Meucci, and P. Ferraro "Optical reconstruction of digital holograms recorded at $10.6 \mu \mathrm{m}$ : route for 3D imaging at long infrared wavelengths” Optics letters 35 (12), 2112-2114 (2010). 